\title{
Triplet diffusion in singlet exciton fission sensitized pentacene solar cells
}

Cite as: Appl. Phys. Lett. 103, 153302 (2013); https://doi.org/10.1063/1.4824420

Submitted: 28 June 2013 • Accepted: 10 September 2013 • Published Online: 08 October 2013

Maxim Tabachnyk, Bruno Ehrler, Sam Bayliss, et al.

openoccess

\section{ARTICLES YOU MAY BE INTERESTED IN}

Solar conversion efficiency of photovoltaic and photoelectrolysis cells with carrier multiplication absorbers

Journal of Applied Physics 100, 074510 (2006); https://doi.org/10.1063/1.2356795

Detailed Balance Limit of Efficiency of p-n Junction Solar Cells

Journal of Applied Physics 32, 510 (1961); https://doi.org/10.1063/1.1736034

Singlet fission efficiency in tetracene-based organic solar cells

Applied Physics Letters 104, 193901 (2014); https://doi.org/10.1063/1.4876600

\section{Challenge us.}

What are your needs for periodic signal detection?

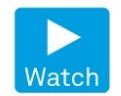

- Zurich

- Instruments

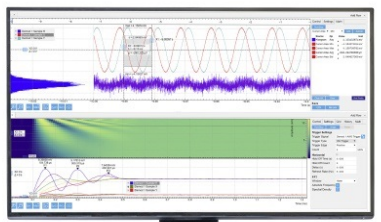

(0 


\title{
Triplet diffusion in singlet exciton fission sensitized pentacene solar cells
}

\author{
Maxim Tabachnyk, Bruno Ehrler, Sam Bayliss, Richard H. Friend, and Neil C. Greenham ${ }^{a}$ \\ Cavendish Laboratory, J. J. Thomson Avenue, University of Cambridge, Cambridge CB3 OHE, \\ United Kingdom
}

(Received 28 June 2013; accepted 10 September 2013; published online 8 October 2013)

\begin{abstract}
Singlet fission sensitized photovoltaics have the potential to surpass the Shockley-Queisser limit for a single-junction structure. We investigate the dynamics of triplet excitons resulting from singlet fission in pentacene and their ionization at a $\mathrm{C}_{60}$ heterojunction. We model the generation and diffusion of excitons to predict the spectral response. We find the triplet diffusion length in polycrystalline pentacene to be $40 \mathrm{~nm}$. Poly(3-hexylthiophene) between the electrode and pentacene works both to confine triplet excitons and also to transfer photogenerated singlet excitons into pentacene with $30 \%$ efficiency. The lower bound for the singlet fission quantum efficiency in pentacene is $180 \pm 15 \%$. (c) 2013 Author(s). All article content, except where otherwise noted, is licensed under a Creative Commons Attribution 3.0 Unported License.

[http://dx.doi.org/10.1063/1.4824420]
\end{abstract}

Fission of photogenerated singlet excitons to pairs of spin-triplet excitons in organic semiconductors such as pentacene provides a promising route to overcome the ShockleyQueisser limit ${ }^{1}$ in photovoltaics. ${ }^{2}$ With an appropriate electron donor/acceptor heterojunction, both triplet excitons can be ionized and therefore used for power conversion. If the second material at the heterojunction is of lower bandgap so that it can harvest low-energy photons, ${ }^{3,4}$ this then allows a theoretical power conversion efficiency of up to $44 \%{ }^{5}$ Singlet fission can be a very rapid process ${ }^{6}$ in materials with a triplet energy less than half the singlet energy, and therefore has the potential to be efficient, although it is generally difficult to quantify triplet yields directly. The long lifetime and associated long diffusion range of triplet excitons allow more straightforward device design as compared to other carrier multiplication processes such as multiple exciton generation. ${ }^{7}$

Transient optical absorption (TA) measurements indicate that singlet excitons in pentacene efficiently undergo fission within $80 \mathrm{fs}$, outcompeting alternative decay mechanisms. ${ }^{6,8}$ High hole mobility ${ }^{9}$ and strong absorption make pentacene an attractive material for singlet fission sensitized solar cells. Using pentacene and the fullerene $\mathrm{C}_{60}$ as the acceptor, Congreve et al. ${ }^{10}$ recently demonstrated a solar cell with external quantum efficiencies (EQE) exceeding 100\%, unambiguously demonstrating efficient singlet exciton fission. A thin layer of poly(3-hexylthiophene) (P3HT) was used in those cells at the hole-collecting indium-tin oxide (ITO) electrode, considerably increasing the photocurrent from pentacene over devices made with ITO alone. We have investigated these device structures and find that the P3HT layer can act as an effective antenna similar to work by Reusswig et al., ${ }^{11}$ transferring singlet excitons to the pentacene layer with $30 \%$ efficiency, and we establish the triplet exciton diffusion range in the pentacene film, noting that previous estimations of the exciton diffusion length in pentacene did not take singlet exciton fission into account. ${ }^{12,13}$

\footnotetext{
${ }^{\text {a) }}$ Author to whom correspondence should be addressed. Electronic mail: ncg11@cam.ac.uk
}

The structure of the pentacene/ $\mathrm{C}_{60}$ donor-acceptor bilayer solar cells studied here is illustrated in Figure 1(a). Following Congreve et al., some of our cells contain a $4 \mathrm{~nm}$ thin P3HT layer. ${ }^{10}$ Details of device fabrication and measurement can be found in Section S1 of the Supplementary Information. ${ }^{14} \mathrm{We}$ calculate the intensity profile of the optical field within the multilayer system using the transfer matrix formalism ${ }^{15,16}$ to estimate the exciton generation profile. This allows the modeling of the EQE assuming 1D diffusion of excitons in pentacene and $\mathrm{C}_{60}$. A fit to the measured $\mathrm{EQE}$ for various cell designs (various pentacene and $\mathrm{C}_{60}$ layer thicknesses, with and without the P3HT layer) allows calculation of the triplet exciton diffusion length $L_{P C}$ and the exciton dissociation efficiency. Moreover, we investigate previous assumptions about the origin of the substantial increase in EQE contribution from pentacene upon the insertion of a P3HT layer. ${ }^{10} \mathrm{We}$ attribute this increase to reflection of triplet excitons at the $\mathrm{P} 3 \mathrm{HT} /$ pentacene interface, and also to transfer of the singlet excitons photogenerated in the P3HT into the pentacene layer. We do not observe a change in hole extraction efficiency from the active layers, in contrast to previous assumptions. ${ }^{10}$

To model the exciton dynamics, we solve the onedimensional diffusion equation (see Supplementary (a)

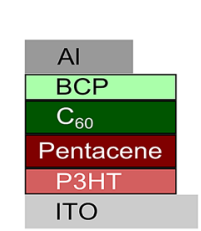

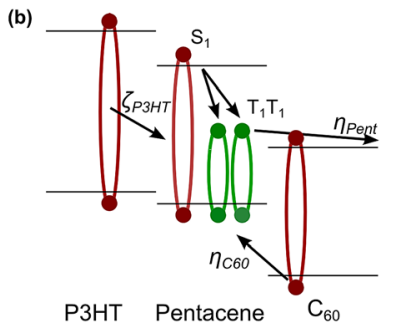

FIG. 1. (a) Device schematic of the solar cells utilizing a pentacene/ $\mathrm{C}_{60}$ donor-acceptor junction, aluminum (Al) and ITO electrodes, and a $10 \mathrm{~nm}$ thin BCP layer. In some cells, a $4 \mathrm{~nm}$ thin P3HT exciton blocking layer was introduced between ITO and the pentacene. (b) In pentacene, the singlet exciton $\left(\mathrm{S}_{1}\right)$ undergoes fission to form two triplet excitons $\left(\mathrm{T}_{1}\right)$, which can be dissociated at the interface with the $\mathrm{C}_{60}$. The efficiency factors for charge injection $\left(\eta_{P e n t}\right.$ and $\left.\eta_{C 60}\right)$ and exciton transfer $\left(\zeta_{P 3 \mathrm{HT}}\right)$ used in the model are indicated. 
Information Section $\mathrm{S}^{14}$ ) in pentacene and $\mathrm{C}_{60}$. The diffusion of singlet excitons in pentacene can be neglected since fission happens on a timescale three orders of magnitude faster than exciton diffusion processes. ${ }^{8}$ For both materials, we use an exciton-extracting pentacene/ $\mathrm{C}_{60}$ interface as one boundary condition. We introduce an empirical factor $\eta_{\text {Pent }}$ for the efficiency of dissociation of pentacene excitons at the interface with $\mathrm{C}_{60}$ (see also Figure 1(b)). Excitons not dissociated are assumed to decay at the interface. Analogously, we define $\eta_{C 60}$ as the efficiency for dissociation of excitons generated in $\mathrm{C}_{60}$.

We assume a singlet-to-triplet fission efficiency of $200 \%$ in pentacene. If the actual value is lower, it will be incorporated in a lower $\eta_{\text {Pent }}$ in the EQE fit. If there is no $\mathrm{P} 3 \mathrm{HT}$ interlayer and pentacene directly contacts ITO, we assume this interface to be exciton absorbing due to a high density of low-energy electronic states available in the metallic ITO. This assumption is validated by the EQE fit below. Where P3HT is present in our solar cells, we define $\zeta_{P 3 H T}$ as the efficiency of excitons generated in P3HT being transferred into pentacene. These transferred singlet excitons are expected to undergo fission in pentacene. We do not perform diffusion modeling in P3HT since the layer is only $4 \mathrm{~nm}$ thick, on the order of the exciton delocalization length. ${ }^{17}$ Noting that the triplet energy in P3HT is $0.6 \mathrm{eV}$ higher than in pentacene, ${ }^{18,19}$ we test the assumption that triplet excitons within the pentacene layer are reflected off the P3HT/pentacene interface due to the lack of (metallic) quenching states. We use the previously measured value of the diffusion length in $\mathrm{C}_{60}$ of 40 $n{ }^{20}$ The bathocuproine (BCP) layer was ignored in the model since AFM images showed that it crystallized in scattered pyramidal structures and covered less than $15 \%$ of the $\mathrm{C}_{60}$ surface.

Apart from the three efficiency parameters, the diffusion length in pentacene is the only free fitting parameter. The efficiency parameters scale the EQE contribution of a material according to its absorption. Since the absorption spectra of pentacene and $\mathrm{C}_{60}$ have little overlap, $\eta_{P e n t}$ and $\eta_{C 60}$ can be fitted independently. In contrast, a change in the diffusion length shifts the $\mathrm{EQE}$ at a wavelength $\lambda$ depending on the exciton generation profile at $\lambda$. Comparing the solar cells with and without a P3HT layer, we can determine $\zeta_{P 3 H T}$ and demonstrate that $\mathrm{P} 3 \mathrm{HT}$ acts as a triplet exciton blocking layer.

The EQE contributions from each of the active materials in a P3HT $(4 \mathrm{~nm}) /$ pentacene $(20 \mathrm{~nm}) / \mathrm{C}_{60}(30 \mathrm{~nm})$ cell are illustrated in Figure 2(a). Fitting the result of the modeling to the measured EQE, we find that $L_{P C}$ has to be larger than $35 \mathrm{~nm}$ to account for the observed enhancement of EQE when the P3HT exciton blocking layer is present, keeping $\eta_{P e n t}$ constant (see Figure 2(b)). This confirms that there is triplet reflection at the pentacene/P3HT interface and the best fit to six cell designs yields $L_{P_{c}}=40 \pm 5 \mathrm{~nm}$. IV curves for two devices and the full set of EQE data and fits can be found in Section S3 of the Supplementary Information. ${ }^{14}$ Since pentacene films have a roughness of up to $3.5 \mathrm{~nm}$ r.m.s. (for $50 \mathrm{~nm}$ layer thickness), we correct the lower error boundary so that $L_{P C}=40_{-9}^{+5} \mathrm{~nm}$. In TA measurements, the triplet lifetime in polycrystalline films of pentacene was determined to be $\tau=5 \mathrm{~ns} .{ }^{8}$ Combined with our value for $L_{P c}, L_{P c}^{2}=D \tau$ yields a diffusion constant $D=3 \times 10^{-3} \mathrm{~cm}^{2} / \mathrm{s}$, which is similar to (a)

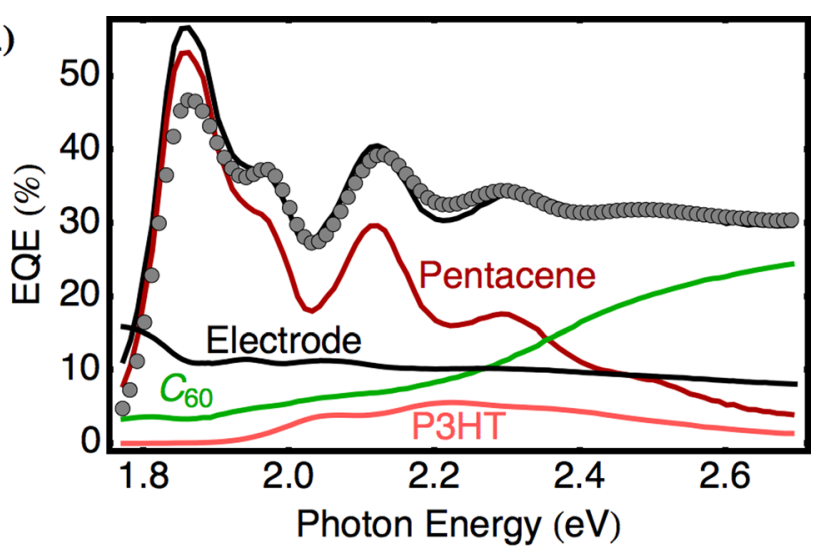

(b)

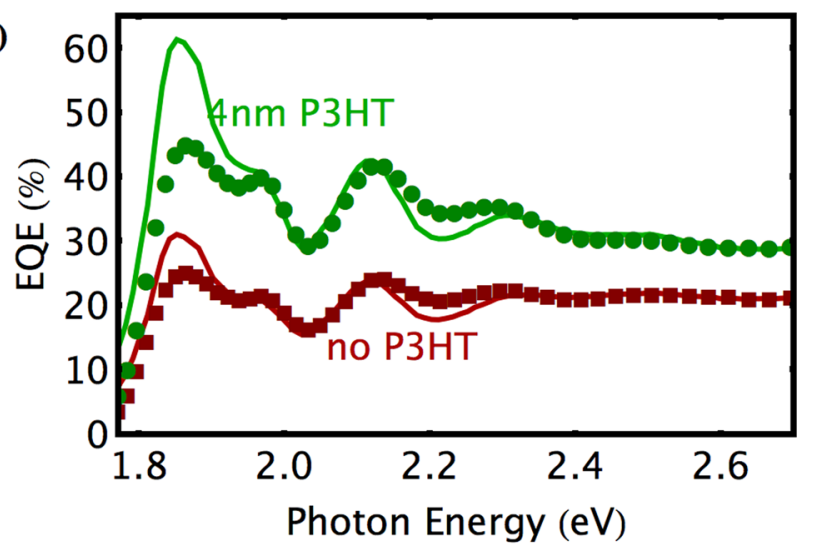

(c)

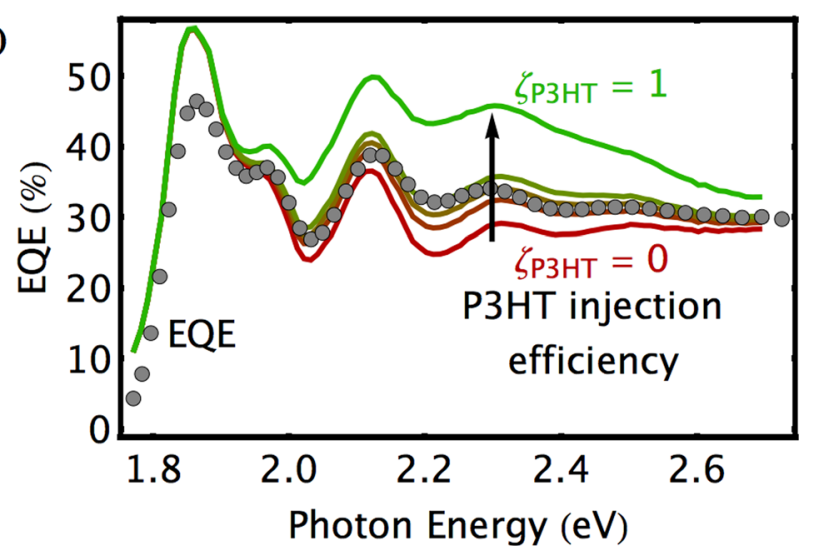

FIG. 2. (a) Measured (-) and modeled (solid line) EQE of a P3HT/pentacene $(20 \mathrm{~nm}) / \mathrm{C}_{60}(30 \mathrm{~nm})$ cell showing the EQE contribution from the active materials and the absorbed light fraction by the electrode. (b) EQE of solar cells made from $50 \mathrm{~nm}$ pentacene and $30 \mathrm{~nm} \mathrm{C} 60$ with $(\bullet)$ and without (ם) $4 \mathrm{~nm}$ P3HT interlayer. The solid lines show the fit to the data. (c) The effect of $\eta_{P 3 H T}$ on the fit to a $\mathrm{P} 3 \mathrm{HT}(4 \mathrm{~nm}) /$ pentacene $(20 \mathrm{~nm}) / \mathrm{C}_{60}(30 \mathrm{~nm})$ cell for $\zeta_{P 3 H T}=(0.0,0.2,0.3,0.4$, and 1.0$)$.

values measured for tetracene. ${ }^{21}$ Our diffusion length is smaller than values previously reported by Kippelen et al. in Refs. 12 and 13 since they had not considered singlet exciton fission.

The influence of exciton transfer from P3HT into pentacene $\left(\zeta_{P 3 H T}\right)$ on the modeled EQE is illustrated in Figure 2(c). The fit yields $\zeta_{P 3 H T}=0.3 \pm 0.1$; presumably this efficiency is limited by singlet exciton quenching at the P3HT/ITO interface as higher values for $\zeta_{P 3 H T}$ have been reported with a poly-(3,4-ethylenedioxythiophene):poly(styrenesulfonate) (PEDOT:PSS) interlayer between P3HT and ITO. ${ }^{10}$ As in Ref. 11, the use of an antenna material can extend the 
photocurrent generated via singlet fission by donating excitons into pentacene. The criteria for an antenna material to donate excitons are: a singlet exciton energy slightly above the singlet exciton energy of pentacene $(1.83 \mathrm{eV}$, Ref. 7$)$ to allow for energy transfer; a triplet exciton energy higher than the triplet exciton energy in pentacene $(0.85 \mathrm{eV}$, Refs. 18 and 22) to avoid triplet transfer; and the highest occupied molecular orbital (HOMO) energy level around $5 \mathrm{eV}$ so as to allow hole extraction across the antenna material. We find that the photocurrent contribution from light absorbed in the $\mathrm{C}_{60}$ layer does not change upon insertion of the P3HT layer. This indicates that the introduction of the P3HT layer does not change the ease of hole extraction as the same fraction of the holes from excitons generated in $\mathrm{C}_{60}$ and dissociated at the pentacene $/ \mathrm{C}_{60}$ interface is extracted from pentacene. (see Figure S10 of the Supplementary Information ${ }^{14}$ )

We find that the measured $J_{S C}$ increases linearly with the intensity up to at least 1 sun illumination intensity and that the EQE is almost unchanged when measured with a whitelight background (see Section S4 of the Supplementary Information ${ }^{14}$ ), indicating that bimolecular processes like triplet-triplet annihilation, triplet-charge annihilation, and interfacial recombination do not limit the cell performance in the relevant intensity regime. We therefore neglect bimolecular effects in the diffusion equation in our EQE model.

Our results for the EQE are consistent with the results of Congreve et al. ${ }^{10}$ when the difference in the distribution of the optical field is taken into account, as explained in detail in Section S5 of the Supplementary Information. ${ }^{14}$ Our model is able to fit the measured EQE with the exception of the lowest-energy pentacene absorption peak $(1.86 \mathrm{eV})$. We note that the ratio of the height of this peak to that of the second peak $(1.95 \mathrm{eV})$ decreases with pentacene thickness. We find a ratio of about 1.1 for the $50 \mathrm{~nm}$ pentacene in agreement with previous studies ${ }^{13,23}$ and about 1.3 for the $20 \mathrm{~nm}$ pentacene, while the $15 \mathrm{~nm}$ pentacene layer studied by Congreve et al. ${ }^{10}$ exhibits a ratio of 1.55 (see also Figure S7 of the Supplementary Information ${ }^{14}$ ). This trend may be due to a lower fission quantum efficiency compared to the higherenergy excitonic states or a less efficient extraction of the fission-generated triplets from lower-energy photons. Due to the distribution of the optical field, low-energy photons are preferentially absorbed closer to the interface between pentacene and ITO and at this interface, the pentacene can stack such that transport is favored in horizontal direction rather than the preferential vertical direction. ${ }^{24}$ In addition, the excitons generated in regions with different stacking directions may have varying dissociation efficiencies as the charge transfer state energy of the pentacene/ $\mathrm{C}_{60}$ molecular couple is minimized for the configuration, where the short molecular axis of pentacene is interfaced with $\mathrm{C}_{60} \cdot{ }^{25}$ Furthermore, because the pentacene crystal diameter in our films $(250 \pm 100 \mathrm{~nm}$, obtained with atomic force microscopy) is comparable to the wavelength of incident light, we may need to consider two separated polarization phases each with its own optical constants and generation profiles. Ellipsometry measurements on pentacene single crystals described in Ref. 26 reveal that the peak at $1.86 \mathrm{eV}$ is mostly excited by a photon polarization parallel to a crystallographic axis $a$ that lies almost in plane with the substrate. The higher-energy peaks are predominantly excited by photon polarizations parallel to a crystallographic axis $b$ almost perpendicular $\left(95^{\circ}\right)$ to $a$. Compared to the generation profiles obtained from polarization-averaged optical constants as used in the rest of our work, $b$-polarized photons generate more high-energy excitons close to the interface to $\mathrm{C}_{60}$ (see Figure S8 of the Supplementary Information ${ }^{14}$ ). Therefore, optical anisotropy of pentacene crystals can qualitatively explain the deviation of the peak at $1.86 \mathrm{eV}$.

For cells with P3HT and thin $(20 \mathrm{~nm})$ pentacene, we obtain $\eta_{C 60}=0.45 \pm 0.02$ and $\eta_{\text {Pent }}=0.90 \pm 0.02$. We assumed that every photon that is absorbed in pentacene generates two excitons. This implies that our measurement for $\eta_{P e n t}$ poses a lower bound for the singlet exciton fission efficiency in pentacene of at least $180 \pm 15 \% . \eta_{\text {Pent }}$ and $\eta_{C 60}$ decrease with increasing layer thickness (see Supplementary Information Table $\mathrm{I}^{14}$ ). Since $\eta_{\text {Pent }}$ describes the dissociation efficiency of pentacene excitons into charges at the pentacene $/ \mathrm{C}_{60}$ interface, losses of excitons at interfacial trap sites provide a possible reason for the dependence of $\eta_{P e n t}$ on layer thickness. The pentacene surface structure changes with layer thickness. Also, Ruiz et al. showed that the orientation of the long axis of pentacene molecules in monolayers is approximately out of the substrate plane for layer thickness of up to $19 \mathrm{~nm} \cdot{ }^{24}$ Density functional theory calculations suggest that the charge transfer state in a pentacene $/ \mathrm{C}_{60}$ molecular couple has its minimum energy for exactly this "head-to-tail" orientation. ${ }^{25}$ Therefore, lower $\eta_{\text {Pent }}$ for 30 and $50 \mathrm{~nm}$ thick layers might be partially due to a change in the interfacial molecular orientation of pentacene.

We obtain the overall internal quantum efficiency (IQE) from the active materials by dividing the measured EQE by the absorbed light fraction in all three active materials. We also computed the IQE from pentacene by accounting for the modeled EQE contributions from $\mathrm{C}_{60}$ and P3HT (see Section S8 of the Supplementary Information ${ }^{14}$ ). The result of this calculation is shown in Figure 3. Both the overall IQE and the pentacene contribution to the IQE exceed $100 \%$. In particular, the IQE for the second Davydov peak $(\approx 2 \mathrm{eV})$ of pentacene reaches $175 \%$. Considering the uncertainties in our model and in the layer thicknesses, we estimate this IQE to be $175 \pm 15 \%$, consistent with complete singlet exciton fission. The dips in the pentacene IQE around $2.1 \mathrm{eV}$ and $2.3 \mathrm{eV}$ can be qualitatively understood as some of the light will have been absorbed into the pentacene-pentacene

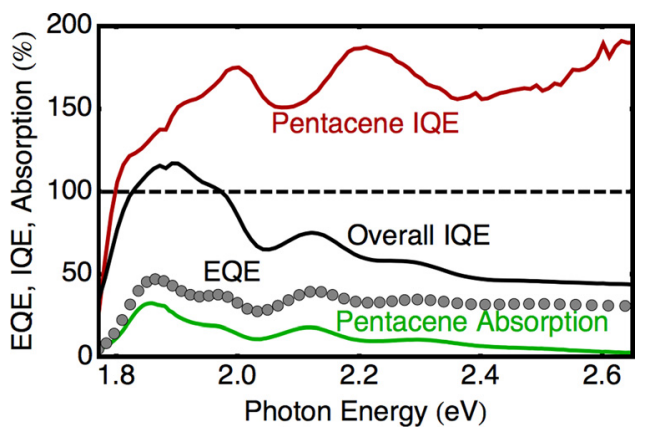

FIG. 3. Measured EQE and modeled absorption in pentacene in a $\mathrm{P} 3 \mathrm{HT} /$ pentacene $(20 \mathrm{~nm}) / \mathrm{C}_{60}(30 \mathrm{~nm})$ cell. Accounting for the modeled EQE contributions of $\mathrm{P} 3 \mathrm{HT}$ and $\mathrm{C}_{60}$ yields the IQE in pentacene. IQE values approaching $200 \%$ are a strong indication of efficient singlet exciton fission. 
charge-transfer $\left(\mathrm{S}_{0} \rightarrow \mathrm{CT}\right)$ states $^{27}$ in this energy regime. These CT states do not undergo fission. ${ }^{8}$

Our studies of the triplet exciton dynamics in pentacene provide valuable insight for the design of singlet fission sensitized solar cells with efficiencies beyond the ShockleyQueisser limit. The lower bound for the singlet exciton fission quantum efficiency is $180 \pm 15 \%$. The diffusion length in pentacene was determined to be $40_{-9}^{+5} \mathrm{~nm}$. Our fit revealed that excitons are injected from $\mathrm{P} 3 \mathrm{HT}$ into pentacene with an absorption-to-injection efficiency of $30 \pm 10 \%$. Therefore, a thin pentacene layer could be used as a fission sensitizer ${ }^{11}$ to split excitons from another highly absorbing material, decoupling absorption from fission. Internal quantum efficiencies approaching $200 \%$ for thin pentacene layers make pentacene a promising candidate for efficient singlet fission sensitized solar cells.

M.T. and B.E. contributed equally to this work. We thank the EPSRC for funding. M.T. acknowledges the Cambridge Gates Trust for financial support. B.E. acknowledges KACST and Selwyn College Cambridge for funding.

${ }^{1}$ W. Shockley and H. J. Queisser, J. Appl. Phys. 32, 510 (1961).

${ }^{2}$ M. B. Smith and J. Michl, Chem. Rev. 110, 6891 (2010).

${ }^{3}$ B. Ehrler, M. W. B. Wilson, A. Rao, R. H. Friend, and N. C. Greenham, Nano Lett. 12, 1053 (2012).

${ }^{4}$ B. Ehrler, K. P. Musselman, M. L. Böhm, R. H. Friend, and N. C. Greenham, Appl. Phys. Lett. 101, 153507 (2012).

${ }^{5}$ M. C. Hanna and A. J. Nozik, J. Appl. Phys. 100, 74510 (2006).

${ }^{6}$ M. W. B. Wilson, A. Rao, J. Clark, R. S. S. Kumar, D. Brida, G. Cerullo, and R. H. Friend, J. Am. Chem. Soc. 133, 11830 (2011).

${ }^{7}$ P. M. Zimmerman, Z. Zhang, and C. B. Musgrave, Nat. Chem. 2, 648 (2010).

${ }^{8}$ A. Rao, M. W. B. Wilson, J. M. Hodgkiss, S. Albert-Seifried, H. Bässler, and R. H. Friend, J. Am. Chem. Soc. 132, 12698 (2010).
${ }^{9}$ T. Hasegawa and J. Takeya, Sci. Technol. Adv. Mater. 10, 24314 (2009).

${ }^{10}$ D. N. Congreve, J. Lee, N. J. Thompson, E. Hontz, S. R. Yost, P. D. Reusswig, M. E. Bahlke, S. Reineke, T. Voorhis, and M. A. Baldo, Science 340, 334 (2013).

${ }^{11}$ P. D. Reusswig, D. N. Congreve, N. J. Thompson, and M. A. Baldo, Appl. Phys. Lett. 101, 113304 (2012).

${ }^{12}$ S. Yoo, B. Domercq, and B. Kippelen, Appl. Phys. Lett. 85, 5427 (2004).

${ }^{13}$ S. Yoo, W. J. Potscavage, Jr., B. Domercq, S.-H. Han, T.-D. Li, S. C. Jones, R. Szoszkiewicz, D. Levi, E. Riedo, S. R. Marder, and B. Kippelen, Solid-State Electron. 51, 1367 (2007).

${ }^{14}$ See supplementary material at http://dx.doi.org/10.1063/1.4824420 for detailed information about the optical constants and modeling and further device characteristics.

${ }^{15}$ Z. Knittl, Optics of Thin Films (Wiley, London, 1976).

${ }^{16}$ L. A. A. Pettersson, L. S. Roman, and O. Inganäs, J. Appl. Phys. 86, 487 (1999).

${ }^{17}$ H. Wang, H.-Y. Wang, B.-R. Gao, L. Wang, Z.-Y. Yang, X.-B. Du, Q.-D. Chen, J.-F. Song, and H.-B. Sun, Nanoscale 3, 2280 (2011).

${ }^{18}$ B. Ehrler, B. J. Walker, M. L. Böhm, M. W. B. B. Wilson, Y. Vaynzof, R. H. Friend, and N. C. Greenham, Nat. Commun. 3, 1019 (2012).

${ }^{19}$ B. Xu and S. Holdcroft, Adv. Mater. 6, 325 (1994).

${ }^{20}$ P. Peumans, A. Yakimov, and S. R. Forrest, J. Appl. Phys. 93, 3693 (2003).

${ }^{21}$ J. B. Aladekomo, S. Arnold, and M. Pope, Phys. Status Solidi B 80, 333 (1977).

${ }^{22}$ J. Burgos, M. Pope, C. E. Swenberg, and R. R. Alfano, Phys. Status Solidi B 83, 249 (1977).

${ }^{23}$ W. J. Potscavage, Jr., S. Yoo, B. Domercq, and B. Kippelen, Appl. Phys. Lett. 90, 253511 (2007).

${ }^{24}$ R. Ruiz, A. C. Mayer, G. G. Malliaras, B. Nickel, G. Scoles, A. Kazimirov, H. Kim, R. L. Headrick, and Z. Islam, Appl. Phys. Lett. 85, 4926 (2004).

${ }^{25}$ P. J. Jadhav, P. R. Brown, N. Thompson, B. Wunsch, A. Mohanty, S. R. Yost, E. Hontz, T. Van Voorhis, M. G. Bawendi, V. Bulovic, and M. A. Baldo, Adv. Mater. 24, 6169 (2012).

${ }^{26}$ M. Dressel, B. Gompf, D. Faltermeier, A. K. Tripathi, J. Pflaum, and M. Schubert, Opt. Express 16, 19770 (2008).

${ }^{27}$ L. Sebastian, G. Weiser, and H. Bässler, Chem. Phys. 61, 125 (1981). 